\title{
Restructuring Tagged Corpora with Morpheme Adjustment Rules
}

\author{
Toshihisa Tashiro Noriyoshi Uratani Tsuyoshi Morimoto \\ ATR Interpreting T'elecommunications Research I aboratories \\ 2-2 Mikaridai, Seika-cho, Soraku-gun, Kyoto 619-02, JAPAN \\ \{tashiro,uratani,morimoto\}@it].atr.co.jp
}

\begin{abstract}
A part-of-speech tagged corpus is a very important knowledge source for natural language processing researchers. 'Today, several part-of-speech tagged corpora are readily available for research use. However, because there is wide diversity of morphological information systems (word-scgmentation, part-of-speech system, etc.), it is difficult to use tagged corpora with an incompatible morphological information system. This paper proposes a method of converting tagged corpora from one morphene system to another.
\end{abstract}

\section{Introduction}

Recently, many natural language processing researchers have concentrated on corpus-based apsproaches. Linguistic corpora can be classified as word-segmented corpora, part-of-speech tagged corpora, and parsed corpora. Because a part-of-speech tagged corpus is the most important corpus, much corpus-based natural language processing research has been performed using part-of-speech tagged corpora.

Ilowever, building a large part-of-speech tagged corpus is very diflicult. It is even more difficult to build a corpus for languages without explicit word boundary characters, such as Japmese. Therefore, researchers always complain of the scarcity of data in the corpus.

'Lo solve this data scarcity problem, previous works proposed methods of increasing the productivity of the labor required for building a part-of-speech tagged corpus. [1].

'This paper proposes anotler method of accuuiring large part-of-speech tagged corpora: restructuring tagged corpora by using morpheme adjustment rules. This method assures good use of the sharable part-of-speech tagged corpora that are already available such as the ATR Dialog Database (ADD) $[2$; 3].

Ideally, these corpora could be used by all researchers and research groups without any modifications. Mowever, actual part-of-speech tagged corpora have the following problems:

- Diversity of orthography:

A word can be spelled in various ways. In Japanese, there are three types of character sets: kanji (漠学), hiragana (ひらが尔), and katakana (カ夕力八). Also, people can use these character sets at their discretion.

- Diversity of word segmentation:

Because the Japanese language has no word boundary characters(i.e. blank spaces), there are no standards of word segmentalion. $\Lambda$ single word in a certain corpus may be considered multiple words in other corpora, and vice versa.

- Diversity of part-ol-speech systems:

There are no standards for part-of-speech systems. It is true that a detailed part-of-speech system can holp the application of part-of-speech information, but the labor required for building corpora will continue to increase. This problem is language-independent.

Diversities of word-segmentation and part-ofspeech systems are fatal problems. The simplest way to solve these problems is to perform a morphological analysis on the raw text in the corpus, with no regard to the word-segmentation and part-of-speech information. Iowever, making a high-quality morphological analyzer demands much time and care. Additionally, it is wasteful to ignore the word-segmentation and part-of-speech information that las been acquired with much eflort.

In restructuring tagged corpora with morpheme adjustment, rules, the worl-segmentation and part-ofspeech information of the original corpus is rewritten, making good use of the original corpus information. This melhod is characterized by reduced manual effort.

In the next section, the method of restructuring tagged corpora is described in detail. Section 3 reports the result of an experiment in rewriting the corpus using this method.

\section{Restructuring Tagged Cor- pora}

Restructuring tagged corpora involves the following three steps:

- preparation of training set

- extraction of morpheme adjustment rules

- rewriting of corpora 


\subsection{Preparation of Training Set}

First, sentences for the training set are chosen from the corpus to be rewritten. New word-segmentation and part-of-speech information (morphological information) is given to the sentences by a morphological analyzer or by hand. Consecuently, the training set has two sets of morphological information for the same raw text. Figure 1 shows an example of the training set.

A large number of training sentences is desirable, but preparing many sentences requires much time and effort. A vast number of sentences would be required to extend coverage to content words (such as nouns, verbs, etc), but functional words (such as particles, auxiliary verbs, etc) can be covered with a smaller number of sentences.

\begin{tabular}{|c|c|}
\hline raw text & 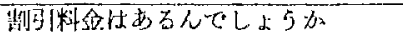 \\
\hline $\begin{array}{l}\text { morpliological } \\
\text { information } A\end{array}$ & 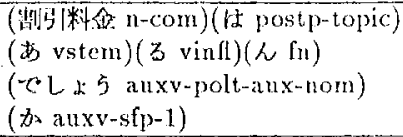 \\
\hline $\begin{array}{l}\text { morphological } \\
\text { in formation } B\end{array}$ & 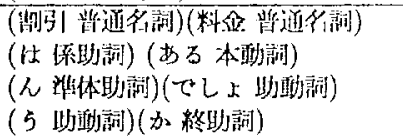 \\
\hline
\end{tabular}

Figure 1: An Example of the Training Set

\subsection{Extraction of Morpheme Adjust- ment Rules}

The method of extracting morpheme adjustment rules from the training set involves finding correspondence between rewriting units and extracting rules for unknown words:

\subsubsection{Correspondences of Rewriting Units}

In languages without explicit word boundary characters, such as Japanese, a single word in a certain morphological information system may be divided into multiple words (one-to-many correspondence) in other morploological information systems, multiple words may be unified (many-to-one correspondence), or the segmentation of multiple words may be changed (many-to-many correspondence). Pigure 2 shows these correspondences.

We developed an algorithrn to find these correspondences (Appendix A). By using this algorithm, morpheme rewriting rules (Figure 3 ) can be extracted.

\subsubsection{Rules for Unknown Words}

Rewriting rules such as those shown in Figure 3 can rewrite only the words that appeared in the training set. If the training set is small, the coverage of the rules will be limited. Ilowever, because this morpheme adjustment is a method of rewriting partof-speech tagged corpora, the treatment of unknown [one-to-one]

"紙 (NOUN)" $\Leftrightarrow$ 川紨 (NOUN)"

[one-to-many]

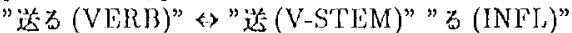

[many-to-one:]

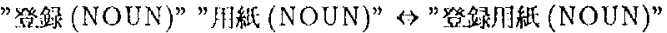

[many-to-many]

"て (PARTICLE)" "いる (VERB)"

$\Leftrightarrow$ "てん(AUXV-STEM)" "る ([NFJ)"

Figure 2: Various correspondences

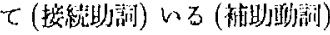

$\Leftrightarrow$ Ch (auxvstem-aspc) $z$ (vinfl)

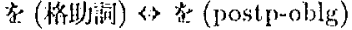

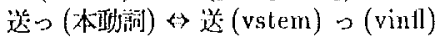

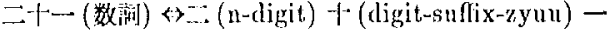

(n-digit)

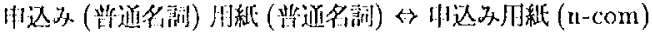

Figure 3: An Example of Extracted Rules

words is easier than with an ordinary morphological analyzer, because that our method can make good use of the part-of-speech information of the original corpus. Rules for unknown words without wordsegmentation changes between two morphological information systems can be extracted automatically from one-to-one correspondence rules in the rewriting rules.

Rules for unknown words with word-segmentation changes can also be extracted automatically by using information concerning the length of the word's characters. For example, when a single verb with two characters in a certain morphological information system corresponds to two words (verb-stem with one character and verb-inflection with one character) in another morphological information system, the following rewriting rule is extracted.

2 (verb) $\rightarrow 1$ (verb-stem) 1(verb-inflection)

Figure 4 shows sample rules for unknown words.

The henristic knowledge of character sets that an ordinary Japanese morphologicat analyzer uses (such as "katakana words are usually proper nouns", "verb inflection words are spelled using hiragana", ete.) are also available in this morpheme adjustment technique.

\subsection{Rewriting of Tagged Corpora \\ 2.3.1 Application of Rewriting Rules}

By applying the rewriting, rules described in the last subsection to the tagged corpus, a lattice structure

格㽖藏 $\Leftrightarrow$ FADN

格助祠 $\leftrightarrow$ POSTP-OBLG

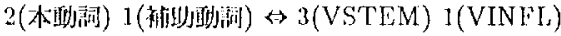

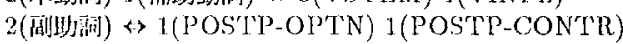

Figure 4: An Lxample of Rules for Unknown Words 


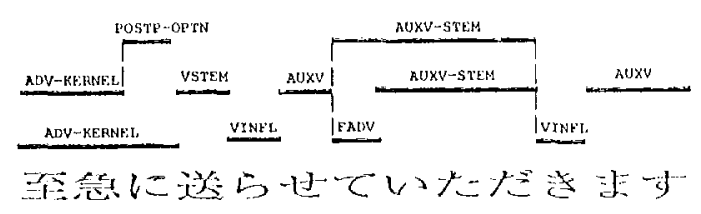

Figure 5: An Fxample of the Jattice Formed by the Morpheme Adjuster

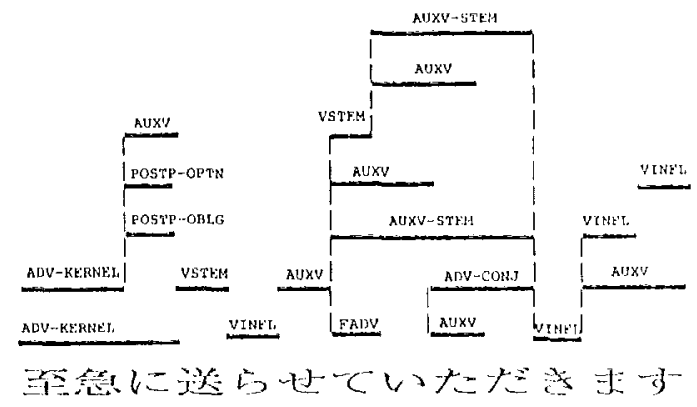

ligure 6: An Example of the Jattice lormed by an Ordinary Morphological Analyzer

(Figure 5) is formed because of the ambignity in rewriting rules. ${ }^{1}$

However, this ambiguity is not as great as the ambiguity that occurs in ordinary morphological analysis because our method makes good use of the informat tion of the original corpus. Figure 6 shows the lattice structure formed when using the ordinary morphological analysis on the same raw text. Note that the size of this latice is greater than the size of the lattice made by our method.

\subsubsection{Lattice Search}

The last step in restructuring tagged corpora can be considered a lattice search problem. In this step, all of the following knowledge sources for ambiguily resolution used in ordinary morphological analysis is also available in our method:

- comection matrix

- henristic preferences (longest word preference, minimum phrase preference, ctc.)

- stochastic preferences (word n-gram, MMM, ete.)

By using these knowledge sources, the most plansible candidate is chosen. In effect, the original corpus is converted to a new corpus that uses a different morphological information system.

\section{Experiment}

\subsection{Fxperimental Condition}

'The targets in our experiment are a morphological information system for the A'TR Jialog Database [2;

\footnotetext{
T.his ambiguity mainly comes from the difference in partof-speech gramblarity between the two morphological information systems.
}

3] and a morphological information system for the unification-based Japanese grammar used in ATR's spoken language parser[4]. These two morphological information systems have the following characterislics.

- The ATR Dialog Database was developed as matterial for analyzing the characteristics of spokenstyle Japanese. 'Therefore, the part-of-speech granularity is coarse. Additionally, because the word segmentation is based on a morphological and etymological criterion, compound nouns and compound words that function as a single auxiliary verb (e.g. "chb") are divided into several shorter word units. On the other hand, because this database gives little consideration to mechanical processing, stems and inflections of inflectional words are not segmented.

- The unilication-based Japanese grammar has a medium-grained part-of-speech (pre-terminal) system to make it both efficient, and easy to maintain[5]. Because the objective of the grammar is to extact the syntactic structures of Japanese sentences automatically and efficiently, componnd words that function as a single word are usually recognized as a single word. On the other hand, stems and inflections of inflectional words are segmented lor convenience of mochan. ical processing.

The above descriptions show that these morphological information systems difler. The objective of this experiment is to examine whether our method can adjust the differences between the two morphological information systems to a considerable extent.

First, we chose 1,000 sentences from the A'l'R Dialog Database as the training set and provicled the morphological information (word-segmentation and part-of-specth) of the mification-based Japanese grammar. We prepared 350 sentences as the test sed, separate from the training set. The test sentences were also given the morphological information.

We extrateded 1,538 correspondences ol rewriting, units (i.e. rewriting rules) and 428 rules for unknown words. These rules can be ased for the bi-directional rewriting experiment.

As the lnowledge source in searching lattices, word bigrans and part-ol-speech bigrams were traned with the traning set. To perform the bi-directional rewriting experiment, these bigrams were trained in both morphological information systems.

'Co compare our' method with ordinary morphological analysis, we developed a simple stochastic morphological analyoe that uses the same bigrams as the knowledge sources ${ }^{2}$. Hecause this morphological analyzer has been developed for the comparative experiment, it cannot manage unknown words. Therefore, the rewriting test was performed by using not only the

\footnotetext{
${ }^{2}$ Of course, the ordinary morphological analyzer can rewrite the corpus much more accurately by using richer knowledge sources. However, it must he noted that our metlod also at use such knowledge sources.
} 


\begin{tabular}{|c|c|c|}
\hline $\begin{array}{c}\text { Morphological } \\
\text { Information }\end{array}$ & $\begin{array}{c}\text { Unification-Based } \\
\text { Japanese Grammar }\end{array}$ & $\begin{array}{c}\text { ATR Dialog } \\
\text { Database }\end{array}$ \\
\hline $\begin{array}{c}\text { Training Set } \\
\text { sentences } \\
\text { (words) }\end{array}$ & $\begin{array}{c}1,000 \\
(10,510)\end{array}$ & $\begin{array}{c}1,000 \\
(10,723)\end{array}$ \\
\hline $\begin{array}{c}\text { Test Set (Full) } \\
\text { sentences } \\
\text { (words) }\end{array}$ & 350 & $\begin{array}{c}350 \\
(4,066)\end{array}$ \\
\hline $\begin{array}{c}\text { Test Set (Sub) } \\
\text { sentences } \\
\text { (words) }\end{array}$ & $(3,894)$ & 148 \\
\hline \hline Vocabulary & $(904)$ & $(949)$ \\
\hline POS System & 1,284 & 1,168 \\
\hline Word Bigram & 75 & 26 \\
\hline POS Bigram & 5,325 & 4,292 \\
\hline
\end{tabular}

Table 1: Experimental Condition

test sentences, but also the training sentences (close experiment) and the sentences having no unknown words (a subset of the test set).

Table 1 shows the experimental conditions in detail.

\subsection{Rewriting of Morphological Infor- mation}

The experiment was performed bi-directionally between the morphological information system of the ATR Dialog Database (ADD) and the morphological information system of unification-based $J$ apanese grammar.

\subsubsection{From Unification-Based Grammar to ADD}

This experiment rewrites from a medium-grained morphological information system to a coarse-grained morphological information system. Table 3.2.1 shows the result of this rewriting. The segmentation error rate and part-of-speech crror rate were calculated using the same definition in [1]. Table 2 shows the result.

The error rates seem to be rather large, but it should be noted that only simple knowledge sources are used both in our method (the morpheme adjuster) and by the morphological analyzer. Also, it is significant that our targets are spoken-style Japanese sentences. Ordinary morphological analyzers can analyze written-style Japanese sentences with a less than $5 \%$ error rate, by using richer knowledge sources[1]. However, previous work reported that the error rate for automatic morphological analysis of the ADD text is more than $15 \%[6]$.

In comparing the two methods, the part-of-specch error rates of our method are clearly better than those of the morphological analyzer. This shows that our method can make good use of the original part-ofspeech information.

\subsubsection{From ADD to Unification-Based Japanese Grammar.}

This experiment is more difficult because this rewriting is from the coarse-grained morphological information system to the medium-grained morphological information system. Table 3 shows the result.

The part-of-speech error rates of our method are better in this rewriting experiment, too.

\section{Conclusion}

This paper proposed restructuring of tagged corpora by using morpheme adjustment rules. The eventual goal of this work is to make precious knowledge sources truly sharable among many researchers. The results of the experiment seem promising.

Our morpheme adjustment method has some resemblance to Brill's part-of-speech tagging method[7]. Brill's simple part-of-speech tagger can be considered a morpheme adjuster that adjusts differences between initial (default) tags and correct tags.

As Brill applied his part-ol-specch tagging technique to the syntactic bracketing technique[8], we believe that our method can be applied to the adjustment of parsed corpora. In the work of Grishman et al.[9], tree rewriting rules to adjust differences between Tree Bank and their grammar were probably prepared manually. By applying our method to parsed corpora, such rewriting rules can be extracted automatically.

\section{Acknowledgments}

The authors would like to thank Dr. Yasuhiro Yamazaki, Presiclent of ATR Lnterpreting Telecommunications Laboratories, for his constant support and encouragement.

\section{References}

[1] Maruyama, Il., Ogino, S., Hidano, M., "The Mega-Word Tagged-Corpus Project," TMI-93, pp.15-23, 1990

[2] Fhara, T., Ogura, K. and Morimoto, T. "ATR Dialogue Database," ICSLP-90, pp.1093-1096, 1990.

[3] Sagisaka, Y., Uratani, N., "ATR Spoken Language Databuse," The Jomnal of the A coustical Society of Japan, Vol. 48, 12, pp. 878-882, 1992. (in Japanese)

[1] Nagala, M. and Morimoto, T.: "A Unification-Based Japanese Parser for Speech-to-Speech 'Lranslation," ITICE 'Trans. Inf. \& Syst, Vol.Er6-D, No.1, pp.51-61, 1993.

[5] Nagata, M. "An Empirical Study on Rule Granularity and Unification Interleaving. - Toward an Efficient UnificationBased Parsing System," in Proc. of COLING-92, 1992.

[6] Kita, K, Ogura, K., Morimoto, T., Yano, Y., "Automatically Fixtracting Frozen Patterns from Corpora Using Cost Criteria,", IPSJ 'Trans. Vol.34, No.9,pp.1937-1943, 1993.(in Japanese)

(7] Brill, E.,: " $\Lambda$ Simple Rule-Based Part of Speech Tagger," Proceedings of the "Third Conference on Applied Natural Language Processing, 1992.

[8] Brill, E., "Automatic Grammar Tntuction and Parsing Free 'Text: "Transformation-Based Lrror-Driven Parsing," ACL.93, 1993.

[9] Rulph Grishmun, Catherine Macleod and John Sterling "Evaluating Parsing Strategies Using Standardized Parse Files," Proceedings of the Third Conference on Applied Natural Janguage Processing, pp.156-161,1992. 


\begin{tabular}{|l|c|c|c|}
\hline Method & segmentation error rate & part-ol-spech error rate & Total \\
\hline Test Set (Full) & $7.8 \%$ & $2.8 \%$ & $10.6 \%$ \\
\hline Test Set (Sub) & $5.1 \%$ & $1.5 \%$ & \\
Morpheme Adjuster & $(6.3 \%)$ & $(3.4 \%)$ & $6.6 \%$ \\
(Morphological Analyzer) & $0.2 \%$ & & $(9.7 \%)$ \\
\hline $\begin{array}{l}\text { Iraining Set(close test) } \\
\text { Morpheme Adjuster } \\
\text { (Morphological Analyzer) }\end{array}$ & $(1.3 \%)$ & $1.5 \%$ & $1.7 \%$ \\
\hline
\end{tabular}

Table 2: Lrom Unification-Based Grammar to ADJ)

\begin{tabular}{|l|c|c|c|}
\hline Method & segnentation error rate & part-ol-spech error rate & Total \\
\hline Test Set (Full) & $8.2 \%$ & $6.9 \%$ & $15.1 \%$ \\
\hline Test Set (Sub) & & $3.1 \%$ & \\
Morpheme Adjuster & $4.2 \%$ & $(6.8 \%)$ & $7.3 \%$ \\
(Morphological Analyzer) & $(8.5 \%)$ & & $(15.3 \%)$ \\
\hline $\begin{array}{l}\text { Training Set (close test) } \\
\text { Morpheme Adjuster } \\
\text { (Morphological Analyzer) }\end{array}$ & $0.5 \%$ & $3.3 \%$ & \\
\end{tabular}

Table 3: From ADD to Unification-Based (inammat

\section{Appendix}

\section{A.The Rule Extraction Algorithm}

type

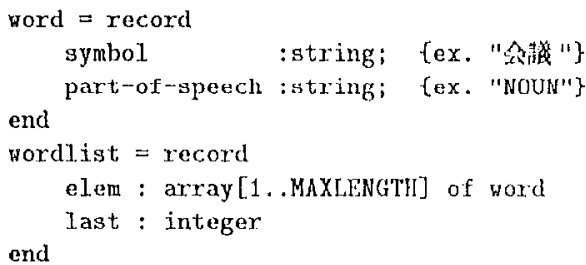

if LFNGTH(111s) = LENGTH (rhs) then begin UUTPUT (Ihs, ths);

cur_a $:=c u r r_{-} a+1 ; \operatorname{cur} b:=c u r_{-} b+1$; Initialize(lhs, rhs);

lhs.elem[1hs.last] := A.e.lem[cur_a];

lhs.last $:=$ Ihs.last +1 ;

rhs.elem[rhs.last] $:=$ B.elem[cur_b];

end

rhs.last $:=$ rhs.last 1 ;

else if LENGIH(lhs) > LENGIH(rhs) then beg cur_b : $=$ cur_b+1;

rhs.elem[rhs.Jast] := D.eleu[cur_b];

rhs. last $:=$ rhs.1ast +1 :

end

else begin

$c u x_{-} a:=c_{2 u r} a+1 ;$

ths, elem[1hs, last] $:=$ A.clem[cur:a]

lhs. 1ast $:=$ lhs. last +1 ;

end

end;

function IENGHL(A: wordList)

[This function returns the total ]ength of

wordlist. Whan the arg is "(ct)

b vstem)(b vinfl))",

this function returns 3.$\}$

var

length, count : integer;

begin

Length $=0$;

for count $:=1$ to $A$. Iast do

length $:=$ length+|A. elem[count] symboll;

return length;

end 